\title{
Primary Glomerulonephritis in Diabetic Patients
}

\author{
Bulent Kaya ${ }^{1}$, Tolga Kuzu${ }^{1}$, Derya Basak Tanburoglu ${ }^{1}$, Saime Paydas ${ }^{1}$, Mustafa Balal ${ }^{1}$, \\ Kivilcim Eren Erdogan ${ }^{1}$, and Gulfiliz Gonlusen ${ }^{1}$ \\ ${ }^{1}$ Cukurova Universitesi Tip Fakultesi
}

July 7, 2020

\begin{abstract}
BACKGROUND Primary glomerulonephritis (PGN) has a significant part in non-diabetic kidney disease (NDKD) in diabetes mellitus (DM) patients. In our study, we compared the clinical, demographic, and laboratory features of patients with biopsyproven diabetic nephropathy (DN) and PGN with type 2 DM METHODS In our retrospective study, type 2 DM patients who underwent kidney biopsy between 2011-2019 were included. Demographic, clinical, and laboratory characteristics of DN and PGN patients were compared. RESULTS Seventy patients with a mean age of $55.7 \pm 9.4$ and $43(61.4 \%)$ males were included. $38(54.3 \%)$ of the patients had DN, and 32 (45.7\%) had PGN. In the PGN, membranous GN (20,62.5\%) was most common. In DN patients, diabetes duration was longer; complications such as retinopathy, neuropathy, hypertension, coronary artery disease, heart failure were more frequent. At the time of renal biopsy, blood sugar, HbA1C, blood pressure, serum albumin, and proteinuria values were similar in 2 groups. The pathological damage findings of kidney biopsy in DN patients were more severe. In the first year after kidney biopsy decrease in eGFR was higher in DN patients, whereas eGFR did not change in PGN patients. CONCLUSION In a diabetic patient, fasting blood sugar, hbA1C, serum albumin, and proteinuria did not differ in the differential diagnosis of DN and PGN, whereas complications of DM (retinopathy, neuropathy, hypertension, coronary artery disease) were more characteristic in differentiation. Detection of PGN in a diabetic patient is crucial for the success of the treatment, according to DN.
\end{abstract}

\section{PRIMARY GLOMERULONEPHRITIS IN DIABETIC PATIENTS}

\section{Background}

Primary glomerulonephritis (PGN) has a significant part in non-diabetic kidney disease (NDKD) in diabetes mellitus (DM) patients. In our study, we compared the clinical, demographic, and laboratory features of patients with biopsy-proven diabetic nephropathy (DN) and PGN with type 2 DM

\section{Methods}

In our retrospective study, type 2 DM patients who underwent kidney biopsy between 2011-2019 were included. Demographic, clinical, and laboratory characteristics of DN and PGN patients were compared.

\section{Results}

Seventy patients with a mean age of $55.7 \pm 9.4$ and $43(61.4 \%)$ males were included. $38(54.3 \%)$ of the patients had DN, and 32 (45.7\%) had PGN. In the PGN, membranous GN (20,62.5\%) was most common. In DN patients, diabetes duration was longer; complications such as retinopathy, neuropathy, hypertension, coronary artery disease, heart failure were more frequent. At the time of renal biopsy, blood sugar, HbA1C, blood pressure, serum albumin, and proteinuria values were similar in 2 groups. The pathological damage findings of kidney biopsy in DN patients were more severe. In the first year after kidney biopsy decrease in eGFR was higher in DN patients, whereas eGFR did not change in PGN patients.

\section{Conclusion}


In a diabetic patient, fasting blood sugar, hbA1C, serum albumin, and proteinuria did not differ in the differential diagnosis of DN and PGN, whereas complications of DM (retinopathy, neuropathy, hypertension, coronary artery disease) were more characteristic in differentiation. Detection of PGN in a diabetic patient is crucial for the success of the treatment, according to DN.

Keywords: Diabetes Mellitus, Diabetic Nephropathy, Glomerular disease, Primary Glomerulonephritis.

\section{What's already known about this topic?}

Diabetic nephropathy is the most common cause of renal involvement in diabetic patients. Non-diabetic kidney disease prevalence may vary depending on renal biopsy selection criteria, and its frequency shows significant variations. Primary glomerulonephritis has a significant part in NDKD in diabetes mellitus patients. The diagnosis of non-diabetic kidney disease is closely related to clinical findings, clinician opinion, and center experience. Besides, its treatment and prognosis are quite different from diabetic nephropathy

\section{What does this article add?}

Fasting blood sugar, hbA1C, serum albumin, and proteinuria did not differ in the differential diagnosis of diabetic nephropathy and primary glomerulonephritis, but diabetic complications, especially diabetic retinopathy, neuropathy, hypertension, coronary artery disease, heart failure were more characteristic in differentiation role diabetic nephropathy from primary glomerulonephritis.

Review criteria: how did you gather, select and analyze the information you considered in your review?

In our study between 2011-2019, 70 diabetic patients older than 18 years were evaluated retrospectively. There were 38 diabetic nephropathies and 32 primary glomerulonephrites. Clinical-demographic characteristics, treatment, and laboratory results of the patients were obtained from the medical records of our hospital. IBM SPSS v. 21 was used as the statistical method

\section{Message for the clinic: what is the 'take-home' message for the clinician?}

In a diabetic patient, primary glomerulonephritis detection is essential due to the successful response to treatment and renal survival, then diabetic nephropathy.

\section{Introduction}

Diabetic Nephropathy (DN) is the most common cause of renal involvement in diabetic patients. It causes renal histopathological changes such as glomerular basement membrane thickening, mesangial matrix increase, diffuse or nodular glomerulosclerosis ${ }^{1}$. Typically, early-stage hyperfiltration develops, followed by microalbuminuria and macroalbuminuria, with slow progressive renal dysfunction. Ultimately, it causes endstage kidney disease (ESRD) ${ }^{2}$. Non-diabetic kidney disease (NDKD) prevalence may vary depending on renal biopsy selection criteria, and its frequency shows significant variations ${ }^{3}$. In renal biopsy samples of diabetic patients, $1 / 3 \mathrm{DN}$ only, $1 / 3 \mathrm{NDKD}$, and $1 / 3 \mathrm{DN}$ and disease have been reported ${ }^{4,5}$. Renal biopsy in diabetic patients is performed in suspicious cases, not routinely ${ }^{6}$. The diagnosis of NDKD is closely related to clinical findings, clinician opinion, and center experience. Besides, its treatment and prognosis are quite different from DN. In a diabetic patient, sudden onset proteinuria, the rapid loss of kidney function, active urinary sediment, and the short-term history of DM may be clues for additional pathologies ${ }^{3,7}$.

Retrospective single-center study, we investigated the clinical, laboratory, and pathological differences of patients with biopsy-proven DN and PGN patients with type 2 DM.

\section{Material and Method}

In our study between 2011-2019, among the 1393 kidney biopsy samples of 80 samples belong to diabetic patients. These 70 diabetic patients older than 18 years were evaluated retrospectively. There were $38 \mathrm{DN}$, $32 \mathrm{PGN}$, and $10 \mathrm{DN}$ with superimposed diseases (such as DN + hypertensive nephropathy, DN + crescentic 
GN) according to kidney biopsy. Ten patients with superimposing conditions with DN were excluded from the study.

Clinical-demographic characteristics, treatment, and laboratory results of the patients were obtained from the medical records of our hospital.

Clinical-demographic characteristics; age, gender, body mass index, smoking, blood pressure, DM duration, accompanying diseases (Hypertension (HT), coronary artery disease (CAD), heart failure), diabetic microvascular complications (diabetic retinopathy (DR), diabetic neuropathy ( DNP)) and drugs used (ACEI (angiotensin-converting enzyme inhibitor), ARB (Angiotensin receptor blocker), oral antidiabetic, Insulin) were evaluated.

At the time of kidney biopsy and follow up period at 6 . and 12 months; biochemical parameters including fasting blood sugar, blood urea nitrogen, creatinine, total protein, albumin, AST, ALT, sodium, potassium, calcium, phosphorus, total cholesterol, HDL cholesterol, LDL cholesterol, triglyceride, complete blood count, $\mathrm{HbA1C}$, proteinuria in 24-hour urine and glomerular filtration rate (eGFR) were recorded. eGFR was calculated according to CKD-EPI ${ }^{8}$. ADA (American Diabetes Association) criteria were used in the diagnosis of type $2 \mathrm{DM}^{9}$. AHA criteria were used for heart failure ${ }^{10}$.

The diagnosis of DR was made by fundoscopy and/or fluorescein angiography, and DNP was made by electromyogram (EMG). Patients using antihypertensive drugs or blood pressure [?] 140/90 were considered hypertensive.

\section{Kidney biopsy and pathological evaluation}

Indications for kidney biopsy in a diabetic patient were active urinary sediment such as dysmorphic erythrocyte, hematuria, erythrocyte, and leucocyte cylinders, the rapid loss of GFR, sudden onset nephrotic syndrome, severe proteinuria, diabetic retinopathy and proteinuria in the absence of other microvascular diseases. A kidney biopsy was performed with a tru-cut needle percutaneously with ultrasonography after obtaining the written consent of the patient.

Kidney biopsy samples were evaluated with light and immunofluorescence (IF). Paraffin-embedded tissues were cut at $3 \mu \mathrm{m}$ for light microscopy evaluation and routine staining. All samples were stained with hematoxylin and eosin (H\&E), Jones methenamine silver, Masson trichrome and periodic acid-schiff, crystal violet, and congo red dyes.

For direct immunofluorescence, the renal tissues were quickly frozen in liquid nitrogen and cut into $3 \mu \mathrm{m}$ sections. In biopsy samples, antibodies against IgG, IgA, IgM, C3, C4, C1q, fibrinogen, kappa, and lambda were examined. All biopsy samples contained at least ten glomeruli. Glomerular basement membrane (GBM) thickening, mesangial expansion, nodular glomerulosclerosis, arteriolar hyalinosis, and arteriosclerosis were evaluated in renal biopsy specimens for the diagnosis of $\mathrm{DN}^{1}$. Specific histopathological diagnostic criteria were used in kidney biopsy of patients with $\mathrm{PGN}^{11}$. Electron microscopy was used for differential diagnosis only in suspected cases.

Chronic damage in the renal parenchyma was evaluated with interstitial fibrosis and tubular atrophy. Renal parenchymal interstitial fibrosis was expressed as a percent and tubular atrophy as yes/no. All biopsy samples were evaluated by experienced nephropathologist.

Ethical Approval All procedures performed in the study were in accordance with the ethical standards of the institutional and/or national research committee and with the 1964 Helsinki Declaration and its later amendments or comparable ethical standards. The ethics committee has approved this study at our university.

\section{Statistical analysis}

IBM SPSS v. 21 (Chicago, IL, USA) was used as the statistical method. Continuous data were expressed as means $\pm \mathrm{SD}$ or median. Categorical variables are given as a number (percentage) of patients. The differences 
between the groups were compared using the chi-square test, Fisher-exact test, Student t-test, and MannWhitney U test. Differences in eGFR among the two groups over time were analyzed using repeated-measures ANOVA. Sphericity was determined by the Mauchly's analysis when the p-value $>0.05$. When the Mauchly's analysis did not identify sphericity, we used repeated-measures ANOVA with Greenhouse-Geisser correction. All statistical tests used the software SPSS version 22.0 (IBM Corp., Armonk, NY, USA). The significance value was accepted as $\mathrm{p}<0.05$.

\section{Results}

In our study, the renal biopsy samples of 70 patients with DM type 2 were evaluated. The mean age was $55.7 \pm 9.4$ of patients 43 (61.4) was male. The pathologic diagnosis of kidney biopsy was $38(54.3 \%) \mathrm{DN}$ and $32(45.7 \%)$ PGN. The average time of DM diagnosis at the time of kidney biopsy was $9.15 \pm 7.5$ years. During the biopsy, two patients were diagnosed with new DM. In PGN group, membranous GN (20,62.5\%), focal segmental glomerulosclerosis (FSGS) $(8,25 \%)$ IgA nephropathy (IgAN) $(2,6.3 \%)$ and minimal change disease $(\mathrm{MDH})(2,6.3 \%)$ were detected (Table 1$)$.

According to PGN in DN patients; the duration of DM was longer $(\mathrm{p}<0.001)$, the frequencies of retinopathy $(\mathrm{p}<0.001)$, neuropathy $(\mathrm{p}<0.001)$, hypertension $(\mathrm{p}=0.0025)$, coronary artery disease $(\mathrm{p}=0.008)$, heart failure $(\mathrm{p}=0.013)$ were higher. In DN group hemoglobin level was lower and the number of patients with eGFR $<30,<60$ and $<90 \mathrm{ml} / \mathrm{min} / 1.73 \mathrm{~m} 2$ were higher (for all $\mathrm{p}<0.001$ ) (Table 2).

At the time of renal biopsy, fasting blood sugar, HbA1C, blood pressure, serum albumin, and proteinuria values were similar in 2 groups. DN patients had lower eGFR values at baseline, sixth and 12th months, and higher proteinuria at sixth and 12th months than PGN patients. In the first year after kidney biopsy decrease in eGFR was higher in DN patients, whereas eGFR did not change in PGN patients. At the time of biopsy, daily proteinuria was not different between the two groups, but in the 6th and 12th months, the daily proteinuria was lower in the PGN group.

The pathological damage findings of kidney biopsy (percentage of interstitial fibrosis, presence of tubular atrophy, sclerosis glomeruli number) in DN patients were more severe.

There were differences for eGFR change between the DN group and PGN group at 0,6 , and 12th months. $(\mathrm{F}(1,56)=46.336, \mathrm{p}<0.001)$ (Table 3 ve Figure 1$)$. There were differences between for eGFR values each other baseline, 6th, and 12th months in DN patients $(\mathrm{F}(1.2,32.392)=16.727, \mathrm{p}<0.001)$. In PGN patients, there was no difference between eGFR changes at 0,6 , and 12th months. $(\mathrm{F}(1.247,7.480)=0.887, \mathrm{p}=0.401)$. There was no difference between eGFR changes at 0,6 , and 12 months in all patients (DN + PGN). (F(1.304, $74.356)=2.388, \mathrm{p}=0.118)$

\section{Discussion}

In our study, we found the frequency of PGN in $45.7 \%$ of 80 diabetic patients. The frequency of NDKD has been reported between $13-82.9 \%^{3}$. Membranous GN was determined most common $(20,62.5 \%)$ among diabetic patients with PGN. This result showed that diabetic patients, especially membranous GN, may play a role in renal injury.

In previous studies, IgAN has been reported to be the most common $\mathrm{GN}^{7,12,13}$. However, membranous GN or other GNs have been reported to be more frequent ${ }^{14-19}$. Since kidney biopsy selection criteria vary according to clinician and center, it is difficult to determine the frequency and cause of NDKD. Many different types of GN can be seen in diabetic patients, such as membranous GN, IgAN, and FSGS.

DR, one of the DM microvascular complications, was detected in $73.6 \%$ of our DN patients, but not in PGN patients. The prevalence of DR in patients with NDKD was reported as $13.6 \%$ and $27.2 \%$, respectively, in the previous two studies ${ }^{20,21}$. As found in our study, the absence of DR can be a predictor of NDKD ${ }^{20,22}$. However, NDKD can be found with $\mathrm{DR}^{23,24}$. Kidney biopsy should be considered in the presence of an atypical scenario, even if a patient with DM has DR. 
The median duration of DM was 11.5 years in DN patients and three years in PGN patients. The duration of DM is closely related to DN. The frequency of microalbuminuria and macroalbuminuria increases after ten years in type $1 \mathrm{DM}^{25}$. In type $2 \mathrm{DM}$, the onset of the disease is difficult to detect, so it is recommended to investigate for DN at the time of diagnosis ${ }^{26}$. As in our study, the incidence of NDKD increased in patients with short diabetes duration ${ }^{27-29}$.

Initial serum albumin, proteinuria, fasting blood sugar, and $\mathrm{HbA1C}$ values were not different in DN and PGN patients. In PGN patients, proteinuria decreased in the 6th and 12 th months with appropriate immunosuppressive therapy, but not in the 12 th month in the DN patients.

In our study, as previously reported studies, initial proteinuria ${ }^{28,30}$ serum albumin $^{19,31,32}$ serum glucose $^{33}$, $\mathrm{HbA1C}^{28,32,34}$ were not different in DN and NDKD patients. Similar to Liu et al. ${ }^{34}$, our DN patients had lower serum hemoglobin levels. This result may be due to our patients with advanced CKD with DN. In our study, similar to previous studies, the mean blood pressure measurements in the two groups were not different ${ }^{21,35}$.

Median eGFR was lower in our DN group at baseline, sixth and 12th months. In addition, DN patients had more evidence of chronic renal damage on kidney biopsy. The median eGFR value decreased at 12 months in DN but did not change in PGN patients. In other words, 1-year renal survival was higher in PGN patients than in the DN group. In previous studies, 5-year renal survival was reported to be better in the NDKD group $^{5,30}$. In this result, the progressive natural course of DN and the successful treatment of PGN patients with appropriate immunosuppressors may play a role.

There were some limitations in our study. These are single-center, retrospective, and insufficient numbers of patients. It is the absence of a standard and exact criteria in the indication of kidney biopsy and consists of biopsy results based on our experience.

As a result, fasting blood sugar, hbA1C, serum albumin, and proteinuria did not differ in the differential diagnosis of DN and PGN, but diabetic complications, especially DR, neuropathy, hypertension, coronary artery disease, heart failure were more characteristic in differentiation role DN from PGN. It can be thought that the frequency of PGN in DM was as much as $45 \%$, and the clinical course was better in patients with PGN so that the biopsy indication may be similar to that of non-diabetic patients. In a diabetic patient, PGN detection is essential due to the successful response to treatment and renal survival, then DN.

\section{Disclosure Statement}

There are no potential conflicts of interest relevant to this article.

\section{Funding Sources}

The authors did not receive any funding

\section{Author Contributions}

Study conception and design: Kaya B, Paydas S, Balal M.

Acquisition of data: all authors

Analysis and interpretation of data: Kaya B, Paydas S, Balal M.

Drafting of the manuscript: Kaya B, Paydas S.

Critical revision: all authors

Kidney biopsies were evaluation: Erdogan KE, Gonlusen G.

\section{REFERENCES}

1. Tervaert TW, Mooyaart AL, Amann K, et al. Pathologic classification of diabetic nephropathy. J Am Soc Nephrol. 2010;21(4):556-563. 
2. Adler AI, Stevens RJ, Manley SE, et al. Development and progression of nephropathy in type 2 diabetes: the United Kingdom Prospective Diabetes Study (UKPDS 64). Kidney Int. 2003;63(1):225-232.

3. Fiorentino M, Bolignano D, Tesar V, et al. Renal biopsy in patients with diabetes: a pooled meta-analysis of 48 studies. Nephrol Dial Transplant. 2017;32(1):97-110.

4. Sharma SG, Bomback AS, Radhakrishnan J, et al. The modern spectrum of renal biopsy findings in patients with diabetes. Clin J Am Soc Nephrol. 2013;8(10):1718-1724.

5. Soleymanian T, Hamid G, Arefi M, et al. Non-diabetic renal disease with or without diabetic nephropathy in type 2 diabetes: clinical predictors and outcome. Ren Fail. 2015;37(4):572-575.

6. Gonzalez Suarez ML, Thomas DB, Barisoni L, Fornoni A. Diabetic nephropathy: Is it time yet for routine kidney biopsy? World J Diabetes. 2013;4(6):245-255.

7. Zhuo L, Ren W, Li W, Zou G, Lu J. Evaluation of renal biopsies in type 2 diabetic patients with kidney disease: a clinicopathological study of 216 cases. Int Urol Nephrol. 2013;45(1):173-179.

8. Levey AS, Stevens LA, Schmid CH, et al. A new equation to estimate glomerular filtration rate. Ann Intern Med. 2009;150(9):604-612.

9. American Diabetes A. 2. Classification and Diagnosis of Diabetes: Standards of Medical Care in Diabetes2018. Diabetes Care.2018;41(Suppl 1):S13-S27.

10. Flack JM, Calhoun D, Schiffrin EL. The New ACC/AHA Hypertension Guidelines for the Prevention, Detection, Evaluation, and Management of High Blood Pressure in Adults. Am J Hypertens.2018;31(2):133135 .

11. KDIGO Clinical Practice Guideline for Glomerulonephritis. 2012.

12. Bi H, Chen N, Ling G, Yuan S, Huang G, Liu R. Nondiabetic renal disease in type 2 diabetic patients: a review of our experience in 220 cases. Ren Fail. 2011;33(1):26-30.

13. Suzuki D, Takano H, Toyoda M, et al. Evaluation of renal biopsy samples of patients with diabetic nephropathy. Intern Med.2001;40(11):1077-1084.

14. Chang TI, Park JT, Kim JK, et al. Renal outcomes in patients with type 2 diabetes with or without coexisting non-diabetic renal disease.Diabetes Res Clin Pract. 2011;92(2):198-204.

15. Kharrat M, Kammoun K, Charfeddine K, et al. [Renal biopsy findings in diabetes mellitus]. Tunis Med. 2007;85(3):216-219.

16. Mazzucco G, Bertani T, Fortunato M, et al. Different patterns of renal damage in type 2 diabetes mellitus: a multicentric study on 393 biopsies. Am J Kidney Dis. 2002;39(4):713-720.

17. Mou S, Wang Q, Liu J, et al. Prevalence of non-diabetic renal disease in patients with type 2 diabetes. Diabetes Res Clin Pract. 2010;87(3):354-359.

18. Pham TT, Sim JJ, Kujubu DA, Liu IL, Kumar VA. Prevalence of nondiabetic renal disease in diabetic patients. Am J Nephrol.2007;27(3):322-328.

19. Dong Z, Wang Y, Qiu Q, et al. Clinical predictors differentiating non-diabetic renal diseases from diabetic nephropathy in a large population of type 2 diabetes patients. Diabetes Res Clin Pract.2016;121:112-118.

20. Wong TY, Choi PC, Szeto CC, et al. Renal outcome in type 2 diabetic patients with or without coexisting nondiabetic nephropathies.Diabetes Care. 2002;25(5):900-905.

21. Soni SS, Gowrishankar S, Kishan AG, Raman A. Non diabetic renal disease in type 2 diabetes mellitus. Nephrology (Carlton).2006;11(6):533-537. 
22. Tone A, Shikata K, Matsuda M, et al. Clinical features of non-diabetic renal diseases in patients with type 2 diabetes.Diabetes Res Clin Pract. 2005;69(3):237-242.

23. Lin YL, Peng SJ, Ferng SH, Tzen CY, Yang CS. Clinical indicators which necessitate renal biopsy in type 2 diabetes mellitus patients with renal disease. Int J Clin Pract. 2009;63(8):1167-1176.

24. Prakash J, Gupta T, Prakash S, et al. Non-diabetic renal disease in type 2 diabetes mellitus: Study of renal - retinal relationship.Indian J Nephrol. 2015;25(4):222-228.

25. Kdoqi. KDOQI Clinical Practice Guidelines and Clinical Practice Recommendations for Diabetes and Chronic Kidney Disease. Am J Kidney Dis. 2007;49(2 Suppl 2):S12-154.

26. American Diabetes Association: Nephropathy in diabetes (Position Statement). Diabetes Care 27 (Suppl.1):S79-S83. 2004.

27. Chong YB, Keng TC, Tan LP, et al. Clinical predictors of non-diabetic renal disease and role of renal biopsy in diabetic patients with renal involvement: a single centre review. Ren Fail.2012;34(3):323-328.

28. Hsieh JT, Chang FP, Yang AH, Tarng DC, Yang CY. Timing of kidney biopsy in type 2 diabetic patients: a stepwise approach. BMC Nephrol. 2020;21(1):131.

29. Liang S, Zhang XG, Cai GY, et al. Identifying parameters to distinguish non-diabetic renal diseases from diabetic nephropathy in patients with type 2 diabetes mellitus: a meta-analysis. PLoS One. 2013;8(5):e64184.

30. Li L, Zhang X, Li Z, et al. Renal pathological implications in type 2 diabetes mellitus patients with renal involvement. J Diabetes Complications. 2017;31(1):114-121.

31. Yenigun EC, Dede F, Ozturk R, et al. Non-Diabetic renal disease in Diabetes Mellitus: clinical features and renal biopsy findings. Hippokratia. 2015;19(2):148-152.

32. Erdogmus S, Kiremitci S, Celebi ZK, et al. Non-Diabetic Kidney Disease in Type 2 Diabetic Patients: Prevalence, Clinical Predictors and Outcomes. Kidney Blood Press Res. 2017;42(5):886-893.

33. Zhuo L, Zou G, Li W, Lu J, Ren W. Prevalence of diabetic nephropathy complicating non-diabetic renal disease among Chinese patients with type 2 diabetes mellitus. Eur J Med Res. 2013;18:4.

34. Liu S, Guo Q, Han H, et al. Clinicopathological characteristics of non-diabetic renal disease in patients with type 2 diabetes mellitus in a northeastern Chinese medical center: a retrospective analysis of 273 cases. Int Urol Nephrol. 2016;48(10):1691-1698.

35. Kritmetapak K, Anutrakulchai S, Pongchaiyakul C, Puapairoj A. Clinical and pathological characteristics of non-diabetic renal disease in type 2 diabetes patients. Clin Kidney J. 2018;11(3):342-347.

\section{TABLES}

Table 1 : Clinical and demographic characteristics of patients $(n=70)$

\begin{tabular}{ll}
\hline Parameters & Mean + SD, $\mathbf{n}(\mathbf{\%})$ \\
\hline Age, years & $55.7 \pm 9.4(26-77)$ \\
Gender, M/F Diagnosis of DM at biopsy time & $43 / 27(61.4 \% / 38,6 \%) 2(\% 2.8)$ \\
BMI, $\mathrm{kg} / \mathrm{m}^{2}$ & $28.1 \pm 4.2(19.5-39.2)$ \\
Smoking & $22(\% 31.4)$ \\
SBP, $\mathrm{mmHg}$ & $131.8 \pm 16.6(100-170)$ \\
DBP, mmHg & $78.4 \pm 9.8(60-100)$ \\
DM duration, year & $9.15 \pm 7.5(0-30)$ \\
HT & $58(\% 82.9)$ \\
CAD & $17(\% 24.3)$ \\
Retinopathy Neuropathy Hyperlipidemia Heart failure & $28(\% 40) 23(\% 32.9) 15(\% 21.4) 7(\% 10)$
\end{tabular}




\begin{tabular}{ll}
\hline Parameters & Mean +SD, n(\%) \\
\hline Oral anti-diabetic Insulin ACEI/ARB & $33(\% 47.1) 37(\% 52.9) 42(\% 60)$ \\
Primary GN DN Membranous GN FSGS IgA Nephropathy MCD & $32(\% 45.7) 38(\% 54.3) 20(\% 62.5) 8(\% 25) 2(\% 6.3) 2(\%$ \\
\hline
\end{tabular}

DN: Diabetic Nephropathy, GN: Glomerulonephritis, SBP: Systolic Blood pressure, DBP: Diastolic Blood pressure, HT: Hypertension, CAD: Coronary artery disease, FSGS: Focal segmental glomerulosclerosis, MCD: Minimal Change Disease, eGFR: Estimated glomerular filtration rate, ACEI: Angiotensin-converting enzyme inhibitory, ARB: Angiotensin receptor blocker

Table 2: Comparison of clinical characteristics diabetic nephropathy and primary glomerulonephritis patients

\begin{tabular}{|c|c|c|c|}
\hline Parameter & $\begin{array}{l}\text { Diabetic Nephropathy } \\
\mathrm{n}=38 \\
\text { median(mean-max) or } \\
\text { mean+ SD }\end{array}$ & $\begin{array}{l}\text { Primary GN } n=32 \\
\text { median }(\text { mean-max) or } \\
\text { mean+ SD }\end{array}$ & $\mathrm{p}$ \\
\hline Male/Female & $22 / 16$ & $21 / 11$ & $0.624^{3}$ \\
\hline Age, year & $55.8 \pm 9.9$ & $55.5 \pm 9.1$ & $0.910^{1}$ \\
\hline BMI, $\mathrm{kg} / \mathrm{m}^{2}$ & $28.1 \pm 4.1$ & $28.1 \pm 4.3$ & $0.967^{1}$ \\
\hline $\mathrm{SBP}, \mathrm{mmHg}$ & $130(100-170)$ & $125(110-170)$ & $0.183^{2}$ \\
\hline $\mathrm{DBP}, \mathrm{mmHg}$ & $80(60-100)$ & $77.5(60-100)$ & $0.075^{2}$ \\
\hline ACEI/ARB & $21 / 17$ & $21 / 11$ & $0.378^{3}$ \\
\hline DM duration, year & $11.5(1-30)$ & $3(0-25)$ & $<0.001^{2}$ \\
\hline Retinopathy, y/n & $28 / 10$ & $0 / 32$ & $<0.001^{3}$ \\
\hline Neuropathy, y/n & $22 / 16$ & $1 / 31$ & $<0.001^{3}$ \\
\hline $\mathrm{CAD}, \mathrm{y} / \mathrm{n}$ & $14 / 24$ & $3 / 29$ & $0.008^{3}$ \\
\hline Heart Failure, y/n & $7 / 31$ & $0 / 32$ & $0.013^{4}$ \\
\hline $\mathrm{HT}, \mathrm{y} / \mathrm{n}$ & $35 / 3$ & $23 / 9$ & $0.025^{3}$ \\
\hline Insulin, $\mathrm{y} / \mathrm{n}$ & $26 / 12$ & $11 / 21$ & $0.004^{3}$ \\
\hline $\mathrm{eGFR}<90$ & $33 / 5$ & $17 / 15$ & $<0.002^{3}$ \\
\hline $\begin{array}{l}\mathrm{ml} / \mathrm{min} / 1.73 \mathrm{~m}^{2}, \mathrm{y} / \mathrm{n} \\
\mathrm{eGFR}<60 \\
\mathrm{ml} / \mathrm{min} / 1.73 \mathrm{~m}^{2}, \mathrm{v} / \mathrm{n}\end{array}$ & $29 / 9$ & $9 / 23$ & $<0.001^{3}$ \\
\hline $\begin{array}{l}\mathrm{ml} / \mathrm{min} / 1.73 \mathrm{~m}^{2}, \mathrm{y} / \mathrm{n} \\
\text { eGFR_0th month, } \\
\mathrm{ml} / \mathrm{min} / 1.73 \mathrm{~m}^{2}\end{array}$ & $37.5(10-116)$ & $88.5(11-127)$ & $<0.001^{2}$ \\
\hline $\begin{array}{l}\text { eGFR_6th month, } \\
\mathrm{ml} / \mathrm{min} / 1.73 \mathrm{~m}^{2}\end{array}$ & $23.5(8-108)$ & $90.5(14-132)$ & $<0.001^{2}$ \\
\hline $\begin{array}{l}\text { eGFR_12th } \\
\text { month,ml } / \min / 1.73 \mathrm{~m}^{2}\end{array}$ & $15(6-105)$ & $89.5(15-141)$ & $<0.001^{2}$ \\
\hline Creatinine, $\mathrm{mg} / \mathrm{dl}$ & $2.02(0.62-4.63)$ & $0.92(0.41-5.14)$ & $<0.001^{2}$ \\
\hline $\mathrm{Hb}, \mathrm{gr} / \mathrm{dl}$ & $10.8 \pm 1.87$ & $13.3 \pm 2.0$ & $<0.001^{1}$ \\
\hline Glucose, mg/dl & $152(60-427)$ & $135(89-290)$ & $0.150^{2}$ \\
\hline $\mathrm{HbA1C}, \%$ & $7.5(5.5-13.7)$ & $6.9(5.5-11)$ & $0.099^{2}$ \\
\hline T.protein, gr/dl & $5.69 \pm 0.93$ & $5.00 \pm 1.21$ & $0.011^{1}$ \\
\hline Albumin, gr/dl & $2.62 \pm 0.63$ & $2.44 \pm 1.04$ & $0.408^{1}$ \\
\hline $\begin{array}{l}\text { Proteinuria_0. month } \\
\text { mg/d }\end{array}$ & $9841 \pm 6052$ & $8778 \pm 4804$ & $0.425^{1}$ \\
\hline $\begin{array}{l}\text { Proteinuria_6. month, } \\
\text { mg/d }\end{array}$ & 6662(874-19467) & $3350(207-13221)$ & $0.003^{2}$ \\
\hline
\end{tabular}




\begin{tabular}{llll}
\hline & $\begin{array}{l}\text { Diabetic Nephropathy } \\
\mathbf{n}=\mathbf{3 8} \\
\text { median(mean-max) } \\
\text { mean+ SD }\end{array}$ & $\begin{array}{l}\text { Primary GN n=32 } \\
\text { median(mean-max) or } \\
\text { mean+ SD }\end{array}$ & \\
Parameter & $10663(750-20500)$ & $1342(102-11575)$ & $\mathrm{p}$ \\
\hline $\begin{array}{l}\text { Proteinuria_12. month, } \\
\text { mg/d }\end{array}$ & $19 / 19$ & $13 / 19$ & $<0.001^{2}$ \\
$\begin{array}{l}\text { Proteinuria }>10 \text { gr/d, } \\
\text { y/n }\end{array}$ & $136.4 \pm 3.1$ & $137.9 \pm 3.8$ & $0.433^{3}$ \\
Na, mmol/l & $8.39 \pm 0.68$ & $8.49 \pm 0.78$ & \\
Ca, mg/dl & $249 \pm 89$ & $289 \pm 82$ & $0.061^{1}$ \\
T. Cholesterol, mg/dl & $203(58-665)$ & $229(91-662)$ & $0.563^{1}$ \\
Triglycerides, mg/dl & $162 \pm 71$ & $191 \pm 70$ & $0.058^{1}$ \\
$\begin{array}{l}\text { LDL, mg/dl } \\
\text { Kidney biopsy Sclerosis } \\
\text { glomeruli count }\end{array}$ & $3(0-16) 10(0-50) 37 / 1$ & $1(0-7) 3(0-20) 23 / 9$ & $0.308^{2}$ \\
$\begin{array}{l}\text { Interstitial fibrosis, } \% \\
\text { Basal membrane }\end{array}$ & $34 / 436 / 2$ & $13 / 1920 / 12$ & $0.088^{1}$ \\
thickening Mesangial & & & $<001^{2}<0.001^{2} 0^{2} 0.004^{3}$ \\
matrix increase & & & \\
Tubular atrophy & & \\
\hline
\end{tabular}

${ }^{1}$ Student T-test, ${ }^{2}$ Mann-Whitney U test, ${ }^{3}$ Chi-square, ${ }^{4}$ Fisher's exact test.

SBP: Systolic Blood pressure, DBP: Diastolic Blood pressure, DM: Diabetes Mellitus, CAD: Coronary artery disease, HT: Hypertension, DM: Diabetes Mellitus, ACEI: Angiotensin-converting enzyme inhibitory, ARB: Angiotensin receptor blocker, eGFR: estimated glomerular filtration rate

Table 3: Post-biopsy 1-year eGFR change in type 2 diabetic patients

\begin{tabular}{lllll}
\hline & eGFR basal & eGFR 6th month & $\begin{array}{l}\text { eGFR 12th } \\
\text { month }\end{array}$ & p \\
\hline $\begin{array}{l}\text { Diabetic } \\
\text { nephropathy }\end{array}$ & $43.8 \pm 30.9$ & $34.07 \pm 27.2$ & $28.3 \pm 27.6$ & $<0.001^{1, \mathrm{~b}}, 0.006^{\mathrm{a}}$, \\
$\begin{array}{l}\text { Primary } \\
\begin{array}{l}\text { Glomerulonephritis } \\
\text { All group }\end{array}\end{array}$ & $82.2 \pm 30.4$ & $86.5 \pm 28.1$ & $87.6 \pm 30.8$ & $0.001^{\mathrm{c}}$ \\
\hline
\end{tabular}

Two-way repeated measured ANOVA test.

${ }^{1}$ Inter groups, ${ }^{\mathrm{a}}$ GFR basal and GFR 6th month, ${ }^{\mathrm{b}}$ GFR basal and GFR 12th month, ${ }^{\mathrm{c}}$ GFR 6th month and GFR 12th month

eGFR: estimated glomerular filtration rate 


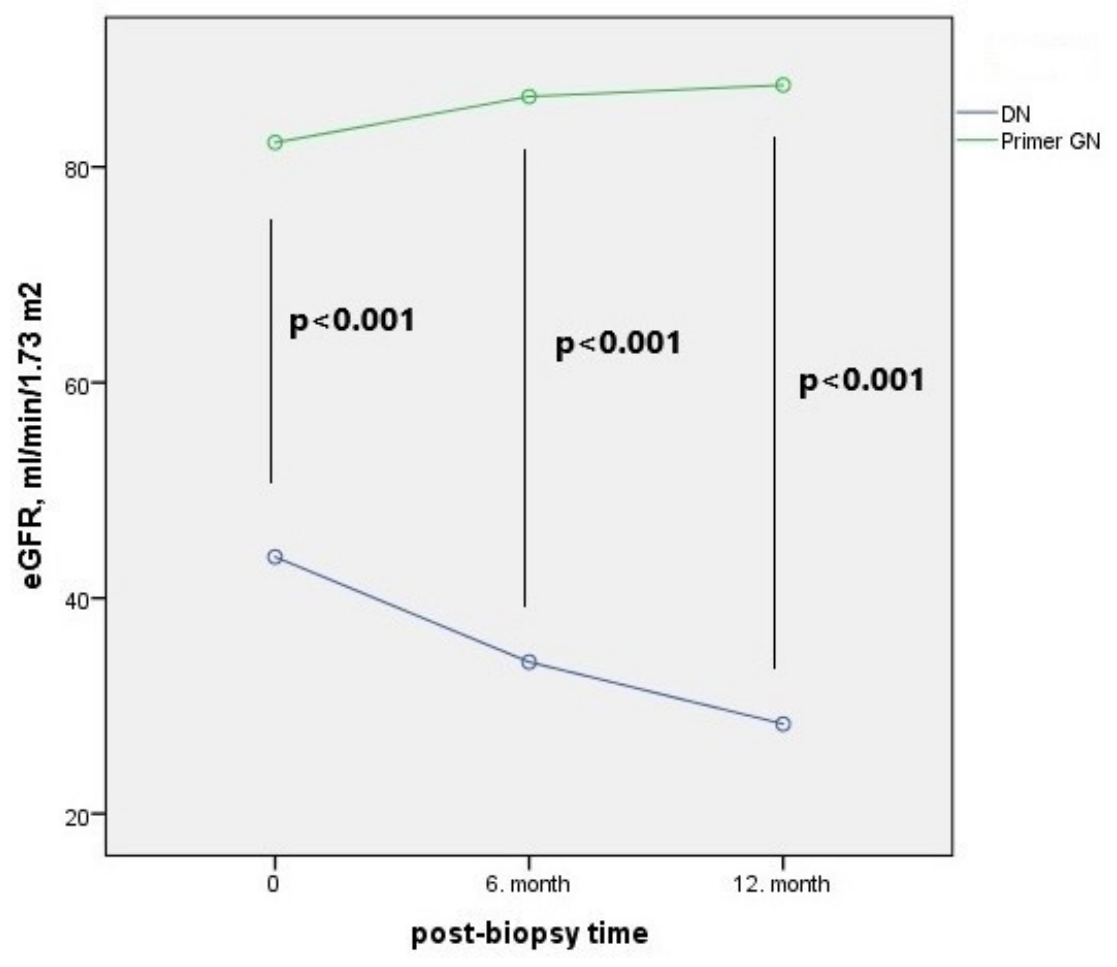

